\title{
Characteristics of Obstructive Sleep Apnea Patients With a Low Body Mass Index: Emphasis on the Obstruction Site Determined by Drug-Induced Sleep Endoscopy
}

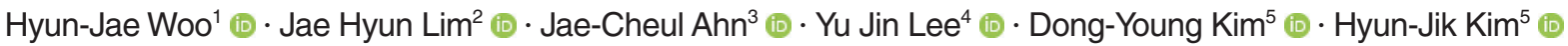 \\ Chae-Seo Rhee ${ }^{5,6}$ (i] $\cdot$ Tae-Bin Won ${ }^{6}$ (i) \\ ${ }^{I}$ Department of Otorhinolaryngology-Head and Neck Surgery, Gumi CHA Hospital, CHA University School of Medicine, Gumi; ${ }^{2}$ Department of \\ Otolaryngology-Head and Neck Surgery, National Police Hospital, Seoul; ${ }^{3}$ Department of Otorhinolaryngology-Head and Neck Surgery, \\ Bundang CHA Hospital, CHA University School of Medicine, Seongnam; Departments of ${ }^{4}$ Psychiatry and ${ }^{5}$ Otorhinolaryngology-Head and Neck \\ Surgery, Seoul National University Hospital, Seoul National University College of Medicine, Seoul; ${ }^{\circ}$ Department of Otorhinolaryngology-Head \\ and Neck Surgery, Seoul National University Bundang Hospital, Seoul National University College of Medicine, Seongnam, Korea
}

Objectives. This study aimed to elucidate the patterns of upper airway collapse in obstructive sleep apnea (OSA) patients with a low body mass index (BMI).

Methods. We designed and conducted a retrospective cohort study. Consecutive patients diagnosed with OSA who underwent drug-induced sleep endoscopy (DISE) were included. Patients were classified into four groups according to their BMI. Age, sex, and polysomnography data were investigated. The patterns of upper airway collapse were characterized by the structures involved (soft palate, tongue base, lateral pharyngeal wall, and epiglottis). We compared the patterns of upper airway collapse in the supine and lateral decubitus position among the four BMI groups using the chi-square test and multivariate binary logistic regression analysis.

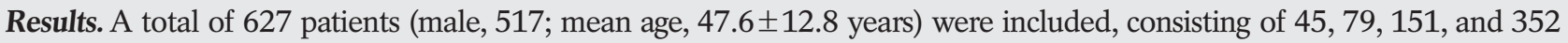
patients who were underweight or lower normal-weight (defined as the low BMI group), upper normal-weight, overweight, and obese, respectively. Severity indicators of OSA, such as the overall apnea-hypopnea index and duration of $\mathrm{SaO}_{2}$ below $90 \%$, were significantly lower in patients with a low BMI than in obese patients, while their average oxygen saturation was significantly higher. The most common obstruction site in the supine position was the tongue base in patients with a low BMI $(100 \%)$, whereas this obstruction site was significantly less common in obese patients $(54.8 \%)$. Tongue base obstruction was mostly relieved in the lateral position, with no discernible obstruction in $86.7 \%$ of the low BMI patients.

Conclusion. Airway obstruction in OSA patients with a low BMI is mostly due to tongue base obstruction, which improves in the lateral position. These characteristics should be kept in mind when considering treatment options for this subgroup of OSA patients.

Keywords. Obstructive Sleep Apnea; Thinness; Body Mass Index; Airway Obstruction; Endoscopy

- Received May 24, 2019

Revised December 18, 2019

Accepted January 2, 2020

- Corresponding author: Tae-Bin Won

Department of Otorhinolaryngology-Head and Neck Surgery, Seoul

National University Bundang Hospital, 82 Gumi-ro 173beon-gil,

Bundang-gu, Seongnam 13620, Korea

Tel: +82-2-2224-2279, Fax: +82-31-787-4057

E-mail: binent@hanmail.net

\section{INTRODUCTION}

Obstructive sleep apnea syndrome (OSAS), which is characterized by repetitive upper airway collapse during sleep, is linked to a multitude of adverse health conditions that decrease quality of life and survival. OSAS is known to be a risk factor, and a pos-

Copyright ( $) 2020$ by Korean Society of Otorhinolaryngology-Head and Neck Surgery.

This is an open-access article distributed under the terms of the Creative Commons Attribution Non-Commercial License (https://creativecommons.org/licenses/by-nc/4.0)

which permits unrestricted non-commercial use, distribution, and reproduction in any medium, provided the original work is properly cited. 
sible causative factor, for the development of systemic hypertension, depression, stroke, angina, and cardiac dysrhythmias [1].

The pathogenesis of obstructive sleep apnea (OSA) is complex, although obesity is considered to be the single most important risk factor for OSA [2]. The impact of obesity on OSAS has been investigated extensively. However, OSAS in underweight patients has not been well characterized, although a recent study showed that underweight OSA patients had a higher apnea-hypopnea index (AHI) and a higher proportion of moderate to severe OSA cases than normal-weight patients [3]. Upper airway changes, such as redundant soft palate, elongated uvula, enlarged tonsils, and oropharyngeal crowding, have been reported to be associated with OSA in obese individuals [4]. However, the anatomical obstruction sites in underweight OSA patients have not been clearly established. A few studies have shown that non-obese OSA patients had concomitant craniofacial skeletal abnormalities, while obese patients had larger upper airway soft tissue structures [5-8].

Drug-induced sleep endoscopy (DISE) has been increasingly used in recent years as a dynamic three-dimensional modality to evaluate upper airway collapse [9]. It has shown to be a valid and reliable method to evaluate the site, degree, and configuration of upper airway obstruction in patients with OSA [10-15]. The aim of the present study was to identify characteristic features of OSA patients with a low body mass index (BMI), with an emphasis on the upper airway obstruction site determined using DISE.

\section{MATERIALS AND METHODS}

This study was approved and informed consent was exempted by the Institutional Review Board of Seoul National University Hospital (IRB No. 1803-111-932).

\section{Study design}

To address the research purpose, we designed and implemented a retrospective cohort study. The study population was composed of the adult patients (age $\geq 19$ years) who visited the sleep cen-

\section{H I G G H L I G G H T S}

- In the supine position, tongue base (TB) obstruction was observed in every patient with a low body mass index (BMI).

- In the supine position, soft palate and lateral pharyngeal wall obstruction was frequently observed in obese patients.

- In the lateral position, TB obstruction showed improvement in most patients irrespective of BMI.

- Airway obstruction in low-BMI obstructive sleep apnea patients is mostly due to TB obstruction, which improves in the lateral position. ter complaining sleep symptoms (such as snoring and/or daytime sleepiness) and underwent nocturnal polysomnography (PSG) and DISE at Seoul National University Hospital (Seoul, Korea) from September 2012 to January 2016. To be included in the study sample, patients had to be diagnosed as OSA with an AHI greater than 5 on full-night attended PSG. Patients who suspected central sleep apnea, overt craniofacial anomaly (defined as craniofacial syndrome which are known to affect airway obstruction, such as trisomy 21, fragile-X syndrome, Treacher Collins syndrome, Prader-Willi syndrome, and Pierre-Robin syndrome) or previous history of upper airway surgery were excluded.

In this study, the primary predictor variables are BMI and sleeping position, and the primary outcomes according to these variables are the obstruction sites of upper airway. The measurement method of each variable is described in the data collection part. Several patients' clinical variables, such as age, sex, AHI (overall, supine, non-supine), $\mathrm{O}_{2}$ saturation (average, lowest, duration of $\mathrm{SaO}_{2}<90 \%$ ), and position dependency, can also influence the primary outcome and are also measured.

\section{Data collection: patients' information}

Subgroup analysis was performed according to BMI. The patients were divided into the following four groups: group 1, the underweight (UW) and lower normal-weight (LNW) group, with a BMI of less than $20.75 \mathrm{~kg} / \mathrm{m}^{2}$; group 2, the upper normal-weight (UNW) group, with a BMI equal to or higher than $20.75 \mathrm{~kg} / \mathrm{m}^{2}$ but less than $23 \mathrm{~kg} / \mathrm{m}^{2}$; group 3, the overweight (OW) group, with a BMI equal to or higher than $23 \mathrm{~kg} / \mathrm{m}^{2}$ but less than $25 \mathrm{~kg} / \mathrm{m}^{2}$; group 4, the obese (OB) group, with a BMI equal to or higher than $25 \mathrm{~kg} / \mathrm{m}^{2}$. Hereafter patients in group 1 are referred to as low BMI OSA patients. Position dependency was determined to be present if the $\mathrm{AHI}$ in the supine position was more than twice the value in the non-supine position [16], only in patients who had more than 30 minutes of lateral sleep time.

\section{Data collection: DISE}

DISE was performed in the following manner as described previously [17]. The patient was positioned comfortably in the supine position. Heart rate and oxygen saturation was monitored throughout the examination, which started in the awake state after unilateral nasal topical anesthesia and decongestion by means of a cotton pledget placed at the middle meatus. Thereafter, sleep was induced by intravenous administration of midazolam (initial dose of $3 \mathrm{mg}$ for adult patients over $50 \mathrm{~kg} ; 0.06 \mathrm{mg} / \mathrm{kg}$ ). After the patient fell asleep, endoscopy (3.8 mm, VNL 1130; Pentax, Tokyo, Japan) was performed through the same nostril. Desaturation events (drop of basal saturation during sleep of more than 3\%) were analyzed for obstruction level, structure, pattern and degree, and representative findings were recorded. If there was no desaturation, changes during snoring were analyzed. An additional bolus of $0.5 \mathrm{mg}$ of midazolam was administered in the event of awakenings. The target level of sedation was upper muscle re- 
laxation-producing obstruction (i.e., snoring or apnea) without respiratory depression. The level of sedation was maintained to a modified Ramsay score of 5 (sluggish response to a light glabellar tap or loud auditory stimulus), or 70 to 80 in selected patients if bispectral index monitoring was instead used.

After observing the airway in the supine position, the patients were positioned in the right lateral decubitus position. The body and head was turned simultaneously so that the axis of the body and the Frankfort plane of the head were perpendicular to the bed. At least three obstructive events were observed in each position, and the most severe event was determined as the obstruction site of the patient. After the conclusion of the examination, $2 \mathrm{mg}$ of flumazenil was administered intravenously as an antidote.

DISE findings were classified according to obstruction structures: soft palate (SP); lateral pharyngeal wall (LPW), including palatine tonsils; tongue base (TB); and epiglottis (EPI). The degree of obstruction was determined as: 0 , no obstruction; 1 , partial obstruction (vibration with desaturation); and 2, complete obstruction (total collapse of airway with desaturation). For obstruction in the $\mathrm{TB}$, more than $50 \%$ displacement compared to the supine awake state was determined as grade 1 , whereas grade 2 obstruction was attributed for more than $75 \%$ obstruction [18]. In this study, we considered a structure to be obstructing when there was grade 2 collapse. The procedures were performed and interpreted by one senior author (TBW).

\section{Data analysis}

Descriptive statistics were computed and values are described as mean \pm standard deviation. Continuous variables (AHI, $\mathrm{O}_{2}$ saturation and duration) were compared by independent $t$-test. Corresponding categorical variables (sex and obstruction site) were compared by chi-square or Fisher's exact test. To determine BMI-specific airway obstruction sites, we used multivariate binary logistic regression analysis. Statistical significance was con- sidered when the $P$-value was $<0.05$. All statistical analyses were performed using IBM SPSS ver. 19.0 (IBM Corp., Armonk, NY, USA).

\section{RESULTS}

\section{Demographic and polysomnographic findings}

The study group comprised 627 patients (517 men and 110 women), with a mean age of $47.6 \pm 12.8$ years. Their average BMI was $26.0 \pm 3.4 \mathrm{~kg} / \mathrm{m}^{2}$. Among the 627 patients, $45(7.2 \%)$ had a BMI of less than $20.75 \mathrm{~kg} / \mathrm{m}^{2}$ and were classified into the UW and LNW group. The 79 patients (12.6\%) whose BMI was between $20.75 \mathrm{~kg} / \mathrm{m}^{2}$ and $23 \mathrm{~kg} / \mathrm{m}^{2}$ were classified as the UNW group, 151 patients $\left(24.1 \%\right.$ ) with a BMI between $23 \mathrm{~kg} / \mathrm{m}^{2}$ and $25 \mathrm{~kg} / \mathrm{m}^{2}$ as the OW group, and 352 patients $(56.1 \%$ ) above $25 \mathrm{~kg} / \mathrm{m}^{2}$ as the OB group. The proportion of women in the UW and LNW group was significantly higher than in the $\mathrm{OB}$ group $(P=0.028)$. The overall AHI, supine AHI, and non-supine AHI of the UW and LNW group were significantly lower than those of the OB group ( $P=0.001, P=0.002$, and $P=0.001$, respectively), but did not show significant differences from the UNW and OW groups. The average oxygen saturation in the UW and LNW group was significantly higher than that in the $\mathrm{OB}$ group $(P=0.020)$, and the duration of oxygen saturation below $90 \%$ was significantly lower than in the OB group $(P=0.012)$ (Table 1$)$. Every OSA patient in the UW and LNW group showed position-dependent OSA, in contrast to the OW and OB groups, in which the proportion was $56.7 \%$ and $24.3 \%$, respectively $(P=0.016$ and $P=0.002$, respectively).

\section{DISE findings according to BMI}

Table 2 presents the obstruction sites according to sleep position in each group during DISE. In the UW and LNW group, the most common structure responsible for obstruction in the supine po-

Table 1. Demographic and clinical characteristics according to body mass index $(n=627)$

\begin{tabular}{|c|c|c|c|c|}
\hline Variable & UW \& LNW $(n=45)$ & UNW $(n=79)$ & OW $(n=151)$ & OB $(n=352)$ \\
\hline Age (yr) & $43.4 \pm 16.8$ & $43.2 \pm 15.1$ & $48.3 \pm 13.4$ & $47.8 \pm 12.3$ \\
\hline Male sex ${ }^{a}$ & $32(71.1)$ & $64(81.1)$ & $120(79.5)$ & $301(85.5)$ \\
\hline Overall $A \mathrm{HI}^{\mathrm{a}-\mathrm{c})}$ & $22.5 \pm 16.8$ & $20.7 \pm 13.7$ & $23.2 \pm 16.2$ & $37.2 \pm 22.9$ \\
\hline Supine $A H I^{a-c, e)}$ & $33.4 \pm 20.7$ & $26.3 \pm 16.4$ & $33.8 \pm 22.5$ & $49.1 \pm 25.7$ \\
\hline Non-supine $A \mathrm{H}^{a-c)}$ & $9.4 \pm 13.8$ & $9.2 \pm 20.2$ & $9.6 \pm 15.8$ & $22.7 \pm 24.1$ \\
\hline Average $\mathrm{SaO}_{2}(\%)^{a-e)}$ & $95.4 \pm 2.8$ & $95.7 \pm 1.3$ & $94.6 \pm 2.8$ & $92.3 \pm 7.0$ \\
\hline Lowest $\mathrm{SaO}_{2}(\%)^{a-c)}$ & $82.6 \pm 8.1$ & $84.9 \pm 6.3$ & $83.6 \pm 7.1$ & $78.9 \pm 9.4$ \\
\hline Duration of $\mathrm{SaO}_{2}<90 \%$ (\% in total sleep $)^{\mathrm{a}-\mathrm{c}}$ ) & $2.9 \pm 7.5$ & $1.9 \pm 7.9$ & $3.3 \pm 9.8$ & $8.2 \pm 15.3$ \\
\hline Position dependency $(\%)^{a-d)}$ & 100 & 66.7 & 56.7 & 24.3 \\
\hline
\end{tabular}

Values are presented as mean \pm standard deviation or number (\%).

UW, underweight $\left(\mathrm{BMl}<18.5 \mathrm{~kg} / \mathrm{m}^{2}\right)$; LNW, lower normal-weight (18.5 $\left.\leq \mathrm{BMl}<20.75 \mathrm{~kg} / \mathrm{m}^{2}\right)$; UNW, upper normal-weight $\left(20.75 \leq \mathrm{BMl}<23 \mathrm{~kg} / \mathrm{m}^{2}\right)$; OW, overweight $\left(23 \leq \mathrm{BMl}<25 \mathrm{~kg} / \mathrm{m}^{2}\right)$; $\mathrm{OB}$, obese $\left(\mathrm{BMI} \geq 25 \mathrm{~kg} / \mathrm{m}^{2}\right)$; $\mathrm{AHI}$, apnea-hypopnea index; $\mathrm{SaO}_{2}$, oxygen saturation.

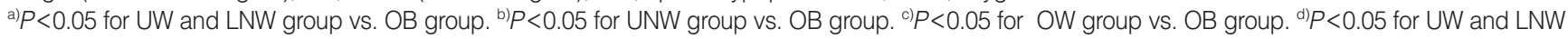
group vs. OW group. ${ }^{e)} P<0.05$ for UNW group vs. OW group. 
Table 2. Obstruction sites according to body mass index $(n=627)$

\begin{tabular}{|c|c|c|c|c|}
\hline Variable & $\begin{array}{c}\text { UW \& LNW } \\
(n=45)\end{array}$ & $\begin{array}{l}\text { UNW } \\
(n=79)\end{array}$ & $\begin{array}{c}\text { OW } \\
(n=151)\end{array}$ & $\begin{array}{c}\mathrm{OB} \\
(n=352)\end{array}$ \\
\hline \multicolumn{5}{|l|}{ Supine } \\
\hline $\mathrm{SP}^{\mathrm{a}, \mathrm{b}, \mathrm{d})}$ & $21(46.7)$ & $46(58.2)$ & $98(64.9)$ & $247(70.2)$ \\
\hline LPW ${ }^{a, d, e)}$ & $7(15.6)$ & $41(51.9)$ & $90(59.6)$ & $224(63.6)$ \\
\hline$T^{a, b, b, d)}$ & $45(100.0)$ & 57 (72.2) & $86(57)$ & $193(54.8)$ \\
\hline EPI & $9(20.0)$ & $21(26.6)$ & $32(21.2)$ & $65(18.5)$ \\
\hline \multicolumn{5}{|l|}{ Lateral decubitus } \\
\hline$S P^{a-d)}$ & $4(8.9)$ & $15(19.0)$ & $39(25.8)$ & $155(44.0)$ \\
\hline$L^{2} W^{a-c)}$ & $3(6.7)$ & $13(16.5)$ & $33(22.2)$ & 197 (55.9) \\
\hline TB & $3(6.7)$ & $7(8.9)$ & $11(7.2)$ & $29(8.2)$ \\
\hline EPI & 0 & $2(2.5)$ & 0 & $1(0.3)$ \\
\hline No obstruction ${ }^{\mathrm{a}, \mathrm{d}-\mathrm{f})}$ & $39(86.7)$ & 27 (34.2) & $42(27.8)$ & $20(5.6)$ \\
\hline
\end{tabular}

Values are presented number (\%). The numbers are not mutually exclusive. UW, underweight $\left(\mathrm{BMI}<18.5 \mathrm{~kg} / \mathrm{m}^{2}\right)$; LNW, lower normal-weight $(18.5 \leq$ $\left.\mathrm{BMl}<20.75 \mathrm{~kg} / \mathrm{m}^{2}\right)$; UNW, upper normal-weight $\left(20.75 \leq \mathrm{BMl}<23 \mathrm{~kg} / \mathrm{m}^{2}\right)$; $\mathrm{OW}$, overweight $\left(23 \leq \mathrm{BMl}<25 \mathrm{~kg} / \mathrm{m}^{2}\right)$; $\mathrm{OB}$, obese $\left(\mathrm{BMI} \geq 25 \mathrm{~kg} / \mathrm{m}^{2}\right) ; \mathrm{SP}$ soft palate; LPW, lateral pharyngeal wall; TB, tongue base; EPI, epiglottis. a) $P<0.05$ for UW and LNW group vs. OB group. ${ }^{\text {b) }} P<0.05$ for UNW group vs. OB group. ${ }^{c} P<0.05$ for $\mathrm{OW}$ group vs. OB group. ${ }^{\mathrm{d}} P<0.05$ for $\mathrm{UW}$ and LNW group vs. OW group. ${ }^{e} P<0.05$ for UNW group vs. OW group. ${ }^{\text {fl }} P<0.05$ for UW and LNW group vs. UNW group.

sition was the TB, as TB obstruction was observed in all patients, whereas the SP, LPW, and EPI contributed to obstruction in $46.7 \%, 15.6 \%$, and $20 \%$ of patients, respectively. There was a trend for an increased frequency of TB obstruction with a decrease in BMI, and the prevalence of TB obstruction in the low $\mathrm{BMI}$ group was significantly higher than in the $\mathrm{OW}$ and $\mathrm{OB}$ groups $(P=0.012$ and $P=0.006$, respectively). Conversely, obstruction of the SP and LPW became more frequent as BMI increased, with the low BMI group having a significantly lower frequency of obstruction at these two sites than the $\mathrm{OW}$ and $\mathrm{OB}$ groups ( $P=0.021$ and $P=0.010$ for SP obstruction, respectively; $P=0.006$ and $P=0.002$ for LPW obstruction, respectively).

In the lateral decubitus position, obstruction of the TB and EPI decreased significantly, irrespective of BMI. The number of patients with no apparent obstruction on DISE was $86.7 \%$ in the low BMI group, which was significantly higher than in other groups. The percentage of patients showing SP and LPW obstruction was $8.9 \%$ and $6.7 \%$, respectively, in the low BMI group; in contrast, in the OW and OB groups, persistent SP and LPW obstruction in $25.8 \%$ and $22.2 \%$ (OW vs. low BMI group; $P=0.011$, $P=0.064$, respectively) and $44 \%$ and $55.9 \%$ (OB vs. low BMI group; $P=0.021, P=0.015$, respectively).

After adjustment for differences by sex, regression analysis was performed to compare the obstruction sites in the UW and LNW group and the OB group (Table 3). The LPW $(P=0.016)$ in the supine position and SP $(P=0.016)$ and LPW $(P=0.014)$ in the lateral decubitus position were obstruction sites that showed significant differences between the UW and LNW group and the OB group. LPW obstruction was less frequent in both the supine position and the lateral decubitus position (odds ratio,
Table 3. Multivariate analysis of the airway obstruction site in the underweight and lower normal-weight group compared to the obese group after sex adjustment

\begin{tabular}{lcc}
\hline Obstruction site & OR (95\% Cl) & $P$-value \\
\hline SP in the supine position & $0.756(0.064-2.616)$ & 0.213 \\
LPW in the supine position & $0.077(0.009-0.624)$ & 0.016 \\
TB in the supine position & $5.72(0.690-47.400)$ & 0.106 \\
SP in the lateral decubitus position & $0.075(0.014-0.616)$ & 0.016 \\
LPW in the lateral decubitus position & $0.072(0.009-0.588)$ & 0.014 \\
\hline
\end{tabular}

$\mathrm{OR}$, odds ratio; $\mathrm{Cl}$, confidence interval; SP, soft palate; LPW, lateral pharyngeal wall; TB, tongue base.

0.077 and 0.075 , respectively).

\section{DISCUSSION}

To our knowledge, this is the first study to evaluate upper airway obstruction patterns in OSA patients with a low BMI using DISE. Previous studies showing anatomical differences between obese and non-obese OSA patients $[5,6,8,19]$ have been limited by the use of static awake lateral cephalograms, a method that falls short of evaluating the dynamic changes in the upper airway during sleep. Those studies have documented that non-obese OSA patients had a significantly narrower distance between the $\mathrm{TB}$ and the posterior pharyngeal wall than non-OSA subjects or obese OSA patients [19]. Recent studies have shown that DISE is a safe, feasible, and valid tool for dynamically assessing the upper airway in OSA patients $[15,17,20,21]$. Sleep position has a major effect on upper airway patency during sleep. It is well known that sleep position affects the occurrence and severity of sleep apnea [22]. In a previous study, we have used DISE to show changes in obstruction sites that occurred when shifting a patient from a supine to lateral decubitus position [17]. Understanding the patterns of obstruction in different postures enables a better characterization of the overall pattern of obstruction in OSA patients. The uniqueness of this study lies in that it evaluated the obstruction sites in OSA patients with a low BMI in different body positions during drug-induced sleep, enabling a more precise characterization of dynamic changes in the upper airway in this group of patients.

The main finding of our study was that the most common structure responsible for obstruction in OSA patients with a low $\mathrm{BMI}$ was the $\mathrm{TB}$, as TB obstruction was present in all patients in the supine position. Interestingly, TB obstruction improved in all but three patients $(6.7 \%)$ when they were changed to a lateral decubitus position. Similarly, the proportion of patients with SP obstruction in the supine position $(46.7 \%)$ decreased to $8.9 \%$ in the lateral decubitus position, and the proportion of those with EPI obstruction dropped from $20 \%$ to $0 \%$. In the majority of low BMI patients, airway obstruction improved when their position was changed from the supine position to the lateral decubi- 
tus position. According to the DISE findings, there was no apparent obstruction in $86.7 \%$ of patients in the lateral position. The structures responsible for airway collapse are usually multiple and inter-related, although the TB plays a major role. The change in posture from the supine position to the lateral decubitus position improves retrodisplacement of the tongue, thereby alleviating TB obstruction, which in turn improves obstruction of the SP and EPI. Although this possibility has not been conclusively established, the improvement of TB obstruction in the lateral decubitus position may be largely related to the effects of gravity on the upper airway [23]. A lack of obstruction in the lateral position during DISE does not guarantee normalization of the AHI, but we have seen a good correlation.

Position-dependent OSA may be related to factors such as disease severity, BMI, age, and sleep stage $[16,24,25]$. When position dependency was evaluated in patients who had a non-supine sleep time of more than 30 minutes, every patient in the low BMI group had position-dependent OSA and in $84 \%$ of these patients, the lateral AHI was lower than 5. Therefore, OSA patients with a low BMI are the best candidates for position therapy, and if successful, position therapy alone can cure most of the patients in this group.

In the multivariate analysis, the LPW in the supine position and the SP and LPW in the lateral decubitus position were statistically less likely to cause obstruction in the UW and LNW group than in the OB group. Although the TB was the most prevalent site of obstruction in the supine position in the low BMI group, TB obstruction did not show a statistically significantly higher prevalence than in the OB group after sex adjustment. A reason for this result may be that TB obstruction was also frequently found in the $\mathrm{OB}$ group. The difference is that $\mathrm{TB}$ obstruction is more likely to coexist with SP and/or LPW obstruction in the OB group [26,27]. We speculate that in the supine position, SP obstruction is masked by TB displacement, causing secondary obstruction of the SP in the low BMI group. When changed to the lateral position, the independent collapsibility of the SP and LPW could be evaluated. Hence, a statistically significant difference was found for SP and LPW collapse in the lateral decubitus position. Patients with severe OSA or those with a higher BMI tend to have higher LPW collapsibility, which causes persistent obstruction in the lateral position [17]. In contrast, airway obstruction in patients with mild OSA or those with a lower BMI is likely to disappear when they change from the supine position to the lateral decubitus position $[22,25,28]$. However, conflicting reports have been published with regard to position-dependent obstruction sites. Although a study showed that patients with SP obstruction were prone to have position-dependent OSA [25], another study implicated retroglossal obstruction as the determinant factor [29]. The characteristic obstruction pattern of the low BMI group can be summarized as universal presence of TB obstruction, with a lack of collapsibility of the SP and LPW.
Keeping these results in mind, the treatment of OSA patients with a low BMI should target TB collapse. Options other than continuous positive airway pressure may include position therapy, the use of oral appliances, and hypoglossal nerve stimulation surgery. Traditional pharyngeal airway surgical procedures, such as uvulopalatopharyngoplasty targeting the SP and or LPW, are not likely to be a good treatment option for most OSA patients with a low BMI.

We adopted an unorthodox method of classifying patients according to their BMI. The number of UW patients according to the WHO classification (BMI less than $18.5 \mathrm{~kg} / \mathrm{m}^{2}$ ) [30] was too small in our cohort $(n=11)$, and the BMI range for the normalweight group $\left(18.5-23 \mathrm{~kg} / \mathrm{m}^{2}\right)$ was judged to be too wide. Therefore, we broke down the normal BMI group into UNW and LNW groups. We believe that combining the UW and LNW groups together enabled a better characterization of those patients whom we intuitively regard as low BMI patients in the clinic. The results of our study showed that this low BMI group, which accounted for $7.2 \%$ of the entire OSA cohort, had a significantly higher proportion of women than the OB group. A large demographic study of 3,659 Japanese OSA patients showed similar results, in that UW patients accounted for $8.6 \%$ of the OSA patients, with a lower male predominance $(77.7 \%)$ than in the obese group (83.3\%) [3].

Traditionally, non-obese OSA patients have been regarded as having milder disease in terms of the AHI [31,32]. However, a thorough comparison of severity of OSA within non-obese patients has not been made. In the previously mentioned cohort study of Japanese OSA patients, the underweight group had a higher AHI than the normal-weight group [3]. The authors suggested that these findings might be unique to Asian OSA patients, but failed to elucidate more specific causes. The results of our study showed that the overall AHI was significantly higher in the OB group compared to the other BMI groups, confirming the results of previous studies. However, when severity was compared among the non-OB groups, the low BMI group did not show significant differences from the other BMI groups. Although a different classification system was used in our study, the severity of OSA in low BMI patients was not significantly different from those in the UW and OW groups. Therefore, it can be misleading to automatically presume that low BMI patients have mild disease.

The limitations of our study include the small number of UW patients, which led us to combine their results together with those of the LNW population. Hence, our findings might not depict the true nature of the UW phenotype. Furthermore, a control group comprising patients with a low BMI who did not have OSA would have been desirable, but would have been difficult to recruit. Soft tissue enlargement may play a more important role in the development of OSA in obese patients, whereas in non-obese patients, bony craniofacial abnormalities may be the dominant contributors to OSA $[5-8,19]$. Although patients 
with overt craniofacial anomalies (syndromic patients) were excluded, more subtle phenotypes such as a retrognathia could have contributed to retroglossal narrowing, partially explaining why the TB was the most common obstruction site in low BMI OSA patients. A further study including a cephalometric analysis and DISE findings would be warranted. There is a concern that the endoscope may affect pharyngeal dynamics. We acknowledge this effect, and have tried to minimize it by using the smallest available endoscope and by performing a stepwise procedure confirming the lower-level obstruction after fully confirming the upper-level obstruction.

In conclusion, OSA patients with a low BMI exhibited distinct characteristics from the higher BMI OSA patients more commonly encountered in clinical settings. Airway obstruction most often occurred due to TB obstruction, which improved in the lateral position. This accounts for the position dependency of these patients and their high likelihood of having a patent airway in the lateral position. These characteristics should be kept in mind when choosing the appropriate treatment for this subgroup of OSA patients. Further studies, including those with a large sample, a normal control group, and cephalometric analysis, are needed in the future.

\section{CONFLICT OF INTEREST}

No potential conflict of interest relevant to this article was reported.

\section{ORCID}

Hyun-Jae Woo

https://orcid.org/0000-0002-3484-0417

Jae Hyun Lim

https://orcid.org/0000-0002-3072-0100

Jae-CheulAhn

https://orcid.org/0000-0002-1191-4209

Yu Jin Lee https://orcid.org/0000-0001-5195-2579

Dong-Young Kim https://orcid.org/0000-0002-4000-1011

Hyun-Jik Kim ～https://orcid.org/0000-0001-8631-928X

Chae-Seo Rhee https://orcid.org/0000-0002-1361-8585

Tae-BinWon ～https://orcid.org/0000-0003-2266-3975

\section{AUTHOR CONTRIBUTIONS}

Conceptualization: HJW, TBW, JCA, YJL. Data curation: HJW, TBW, JHL, JCA. Formal analysis: HJW, TBW, JCA. Funding acquisition:TBW, DYK, HJK, CSR. Methodology: HJW, TBW, JHL, YJL. Project administration: HJW,TBW.Visualization: HJW.Writing-original draft: HJW. Writing-review \& editing: TBW, JHL, DYK, HJK, CSR.

\section{REFERENCES}

1. Ulualp SO. Snoring and obstructive sleep apnea. Med Clin North Am. 2010 Sep;94(5):1047-55.

2. Young T, Peppard PE, Gottlieb DJ. Epidemiology of obstructive sleep apnea: a population health perspective. Am J Respir Crit Care Med. 2002 May;165(9):1217-39.

3. Ohdaira F, Nakamura K, Nakayama H, Satoh M, Ohdaira T, Nakamata M, et al. Demographic characteristics of 3,659 Japanese patients with obstructive sleep apnea-hypopnea syndrome diagnosed by full polysomnography: associations with apnea-hypopnea index. Sleep Breath. 2007 Jun;11(2):93-101.

4. Friedman M, Tanyeri H, La Rosa M, Landsberg R, Vaidyanathan K, Pieri S, et al. Clinical predictors of obstructive sleep apnea. Laryngoscope. 1999 Dec;109(12):1901-7.

5. Sakakibara H,Tong M, Matsushita K, Hirata M, KonishiY, Suetsugu S. Cephalometric abnormalities in non-obese and obese patients with obstructive sleep apnoea. Eur Respir J. 1999 Feb;13(2):403-10.

6. Nelson S, Hans M. Contribution of craniofacial risk factors in increasing apneic activity among obese and nonobese habitual snorers. Chest. 1997 Jan;111(1):154-62.

7. Tsuchiya M, Lowe AA, Pae EK, Fleetham JA. Obstructive sleep apnea subtypes by cluster analysis. Am J Orthod Dentofacial Orthop. 1992 Jun;101(6):533-42.

8. Ferguson KA, OnoT, Lowe AA, Ryan CF, Fleetham JA.The relationship between obesity and craniofacial structure in obstructive sleep apnea. Chest. 1995 Aug;108(2):375-81.

9. Aktas O, Erdur O, Cirik AA, Kayhan FT. The role of drug-induced sleep endoscopy in surgical planning for obstructive sleep apnea syndrome. Eur Arch Otorhinolaryngol. 2015 Aug;272(8):2039-43.

10. Sadaoka T, Kakitsuba N, FujiwaraY, Kanai R,Takahashi H.The value of sleep nasendoscopy in the evaluation of patients with suspected sleep-related breathing disorders. Clin Otolaryngol Allied Sci. 1996 Dec;21(6):485-9.

11. Steinhart H, Kuhn-Lohmann J, Gewalt K, Constantinidis J, Mertzlufft F, Iro H. Upper airway collapsibility in habitual snorers and sleep apneics: evaluation with drug-induced sleep endoscopy. Acta Otolaryngol. 2000 Oct;120(8):990-4.

12. Berry S, Roblin G,Williams A, Watkins A, Whittet HB.Validity of sleep nasendoscopy in the investigation of sleep related breathing disorders. Laryngoscope. 2005 Mar;115(3):538-40.

13. Rabelo FA, Braga A, Kupper DS, De Oliveira JA, Lopes FM, de Lima Mattos PL, et al. Propofol-induced sleep: polysomnographic evaluation of patients with obstructive sleep apnea and controls. Otolaryngol Head Neck Surg. 2010 Feb;142(2):218-24.

14. Rodriguez-Bruno K, Goldberg AN, McCulloch CE, Kezirian EJ.Testretest reliability of drug-induced sleep endoscopy. Otolaryngol Head Neck Surg. 2009 May;140(5):646-51.

15. Kezirian EJ, White DP, Malhotra A, Ma W, McCulloch CE, Goldberg AN. Interrater reliability of drug-induced sleep endoscopy. Arch Otolaryngol Head Neck Surg. 2010 Apr;136(4):393-7.

16. Oksenberg A, Silverberg DS, Arons E, Radwan H. Positional vs nonpositional obstructive sleep apnea patients: anthropomorphic, nocturnal polysomnographic, and multiple sleep latency test data. Chest. 1997 Sep;112(3):629-39.

17. Lee CH, Kim DK, Kim SY, Rhee CS, Won TB. Changes in site of obstruction in obstructive sleep apnea patients according to sleep position: a DISE study. Laryngoscope. 2015 Jan;125(1):248-54.

18. Hohenhorst W, Ravesloot MJ, Kezirian EJ, de Vries N. Drug-induced sleep endoscopy in adults with sleep-disordered breathing: technique and theVOTE classification system. OperTech Otolayngol Head Neck Surg. 2012 Mar;23(1):11-8.

19. Tangugsorn V, Krogstad O, Espeland L, Lyberg T. Obstructive sleep 
apnoea: multiple comparisons of cephalometric variables of obese and non-obese patients. J Craniomaxillofac Surg. 2000 Aug;28(4): 204-12.

20. Kezirian EJ, Hohenhorst W, de Vries N. Drug-induced sleep endoscopy: the VOTE classification. Eur Arch Otorhinolaryngol. 2011 Aug; 268(8):1233-6.

21. Lee CH, Hong SL, Rhee CS, Kim SW, Kim JW.Analysis of upper airway obstruction by sleep videofluoroscopy in obstructive sleep apnea: a large population-based study. Laryngoscope. 2012 Jan;122(1): 237-41.

22. Cartwright RD. Effect of sleep position on sleep apnea severity. Sleep. 1984;7(2):110-4.

23. Harper R, Sauerland EK. The role of the tongue in sleep apnea. In: Guilleminault C, Dement WC, editors. Sleep apnea syndromes. New York (NY): Alan R. Liss; 1978. p. 219-34.

24. Mador MJ, Choi Y, Bhat A, Dmochowski J, Braun M, Gottumukkala VA, et al. Are the adverse effects of body position in patients with obstructive sleep apnea dependent on sleep stage? Sleep Breath. 2010 Feb;14(1):13-7.

25. RichardW, Kox D, den Herder C, Laman M, van Tinteren H, deVries $\mathrm{N}$. The role of sleep position in obstructive sleep apnea syndrome. Eur Arch Otorhinolaryngol. 2006 Oct;263(10):946-50.

26. Ravesloot MJ, de Vries N. One hundred consecutive patients under- going drug-induced sleep endoscopy: results and evaluation. Laryngoscope. 2011 Dec;121(12):2710-6.

27. Abdullah VJ, Wing YK, van Hasselt CA. Video sleep nasendoscopy: the Hong Kong experience. Otolaryngol Clin North Am. 2003 Jun; 36(3):461-71.

28. Itasaka Y, Miyazaki S, Ishikawa K, Togawa K. The influence of sleep position and obesity on sleep apnea. Psychiatry Clin Neurosci. 2000 Jun;54(3):340-1.

29. Sunwoo WS, Hong SL, Kim SW, Park SJ, Han DH, Kim JW, et al. Association between positional dependency and obstruction site in obstructive sleep apnea syndrome. Clin Exp Otorhinolaryngol. 2012 Dec;5(4):218-21.

30. World Health Organization. The Asia-Pacific perspective: redefining obesity and its treatment. Sydney: Health Communications Australia; 2000.

31. Chirakalwasan N,Teerapraipruk B, Simon R, Hirunwiwatkul P, Jaimchariyatam N, Desudchit T, et al. Comparison of polysomnographic and clinical presentations and predictors for cardiovascular-related diseases between non-obese and obese obstructive sleep apnea among Asians. J Clin Sleep Med. 2013 Jun;9(6):553-7.

32. Ralls FM, Grigg-Damberger M. Roles of gender, age, race/ethnicity, and residential socioeconomics in obstructive sleep apnea syndromes. Curr Opin Pulm Med. 2012 Nov;18(6):568-73. 